PROCEEDINGS OF THE

AMERICAN MATHEMATICAL SOCIETY

Volume 138, Number 12, December 2010, Pages 4405-4411

S 0002-9939(2010)10437-9

Article electronically published on June 10, 2010

\title{
SUB-BERGMAN SPACES IN THE UNIT BALL OF $\mathbb{C}^{n}$
}

\author{
FRÉDÉRIC SYMESAK
}

(Communicated by Franc Forstneric)

\begin{abstract}
Let $\Phi(z)=\left(\varphi_{1}(z), \cdots, \varphi_{l}(z)\right)$ be holomorphic from the unit ball of $\mathbb{C}^{n}$ into the unit ball of $\mathbb{C}^{l}$. We denote by $B_{\alpha}(z, w)$ the weighted Bergman kernel. We give a condition for the kernel $(1-\Phi(z) \overline{\Phi(w)}) B_{\alpha}(z, w)$ to be a reproducing kernel and we study the related Hilbert space.
\end{abstract}

\section{INTRODUCTION}

The aim of this note is to give a characterization of some reproducing kernels of spaces of holomorphic functions on the unit ball of $\mathbb{C}^{n}$. These kernels are obtained from the weighted Bergman kernel $B_{\alpha}(z, w)$ and bounded holomorphic functions $\varphi_{1}(z), \cdots, \varphi_{l}(z)$.

We denote by $\mathbb{B}$ the unit ball of $\mathbb{C}^{n}$ and by $r(z)=\sum_{i=1}^{n}\left|z_{i}\right|^{2}-1$ a defining function. Let $\alpha>-1$ and let $d V_{\alpha}(z)=(-r(z))^{\alpha} d V(z)$. We denote by $\mathbb{A}_{\alpha}^{2}$ the weighted Bergman space of $\mathbb{B}$ with respect to the measure $d V_{\alpha}(z)[5]$. It is a Hilbert space and its reproducing kernel is the weighted Bergman kernel $B_{\alpha}(z, w)=$ $\frac{c_{\alpha}}{(1-z \bar{w})^{n+1+\alpha}}$. Let $\Phi(z)=\left(\varphi_{1}(z), \cdots, \varphi_{l}(z)\right)$ be holomorphic functions on the unit ball. We consider the kernel

$$
K_{\alpha}(z, w)=(1-\Phi(z) \overline{\Phi(w)}) B_{\alpha}(z, w) .
$$

We have the following result:

Theorem 1.1. Let $\alpha>-1$ and let $\Phi(z)=\left(\varphi_{1}(z), \cdots, \varphi_{l}(z)\right)$ be holomorphic. The kernel $K_{\alpha}(z, w)$ is a reproducing kernel if and only if $\sup _{\mathbb{B}}|\Phi(w)| \leq 1$.

Let $\varphi$ be a holomorphic function; the reproducing property of kernels of type $(1-\varphi(z) \overline{\varphi(w)}) B(z, w)$ was studied by F. Beatrous and J. Burbea 2 for spaces of holomorphic functions on domains of $\mathbb{C}^{n}$ and by S. Saitoh for abstract kernels of Hilbert spaces of functions [7. In the case of the unit disc of $\mathbb{C}$, when $\varphi$ is a finite Blaschke product, the characterization of the related Hilbert space was obtained by K. Zhu in [10] and [11] for Bergman spaces and by S. Sultanic for weighted Bergman spaces [9. The same question was introduced by L. De Branges and J. Rovnyak in the context of Hardy spaces [8]. The following theorem gives the Hilbert space associated to the kernel $K_{\alpha}(z, w)$ in the case of the unit ball of $\mathbb{C}^{n}$.

Received by the editors November 3, 2009 and, in revised form, February 10, 2010.

2010 Mathematics Subject Classification. Primary 32A36; Secondary 46E22.

Key words and phrases. Bergman spaces, sub-Hilbert spaces, reproducing kernel. 
For $-n \leq \alpha<-1, B_{\alpha}(z, w)$ is the reproducing kernel of the diagonal weighted Besov space $\mathcal{B}_{\alpha}^{2}$. A holomorphic function $f$ belongs to $\mathcal{B}_{\alpha}^{2}$ if and only if $(I+R)^{m} f$ is in $\mathbb{A}_{2 m+\alpha}^{2}$, where $R=\sum_{k=1}^{n} z_{k} \partial_{z_{k}}$ is the radial derivative and $m>(-\alpha-1) / 2$ [13. In this case we have the following:

Theorem 1.2. Let $\Phi(z)=\left(\varphi_{1}(z), \cdots, \varphi_{l}(z)\right)$ be holomorphic and non-constant. The functional Hilbert space associated to $K_{\alpha}(z, w)$ is, with an equivalent norm,

(1) $\mathbb{A}_{\alpha-1}^{2}$ when $\alpha>0$,

(2) the Hardy space $\mathbb{H}^{2}$ when $\alpha=0$,

(3) $\mathcal{B}_{\alpha-1}^{2}$ when $-1<\alpha<0$

if and only if there exists $C>0$ such that $1-|\Phi(z)|^{2} \leq C(-r(z))$ for $z$ in $\mathbb{B}$.

Let us mention an example with $n=l \geq 2$ which motivates this note. Let $a$ be a point of $\mathbb{B}_{n}$ and denote by $\Phi_{a}$ the related holomorphic automorphism. A straightforward computation gives $K_{\alpha}(z, w)=\frac{c B_{\alpha-1}(z, w)}{(1-z \bar{a})(1-a \bar{w})}$ and the Hilbert space is $\mathbb{A}_{\alpha-1}^{2}$ equipped with the norm $\|f\|^{2}=\int_{\mathbb{B}}|f(w)|^{2}|1-a \bar{w}|^{2} d V_{\alpha-1}(w)$.

In the case where $a=0$, the kernel is $B_{\alpha-1}(z, w)$ and the Hilbert space is $\mathbb{A}_{\alpha-1}^{2}$ with its standard norm.

As far as we know, automorphisms are the only applications which satisfy the condition of the theorem in the case where $n=l \geq 2$.

\section{Sub-Bergman spaces}

Recall that the Bergman projection $B_{\alpha}$ is the orthogonal projection from $L^{2}\left(d V_{\alpha}\right)$ onto $\mathbb{A}_{\alpha}^{2}$. Given a bounded function $\varphi$, the Toeplitz operator of symbol $\varphi$ is defined by $T_{\varphi} f=B_{\alpha}(\varphi f)$. We consider the positive operator $H$ given as follows:

Definition 2.1. Let $\Phi(z)=\left(\varphi_{1}(z), \cdots, \varphi_{l}(z)\right)$ be holomorphic. We set

$$
H f=\sum_{i=1}^{l} \varphi_{i} T_{\bar{\varphi}_{i}} f, f \in \mathbb{A}_{\alpha}^{2} .
$$

We first calculate the norm of $H$.

Proposition 2.2. Let $H$ be as above. Then $\|H\|=\sup _{\mathbb{B}}|\Phi(w)|^{2}$.

Proof. Let $f$ be in $\mathbb{A}_{\alpha}^{2}$. Then

$$
\|H f\|_{\mathbb{A}_{\alpha}^{2}} \leq \sum_{i=1}^{l}\left\|\varphi_{i} B_{\alpha}\left(\overline{\varphi_{i}} f\right)\right\|_{\mathbb{A}_{\alpha}^{2}} \leq \sup _{\mathbb{B}}|\Phi(w)| \sum_{i=1}^{l}\left\|B_{\alpha}\left(\overline{\varphi_{i}} f\right)\right\|_{\mathbb{A}_{\alpha}^{2}}
$$

Since $B_{\alpha}$ is a projection, $\left\|B_{\alpha}\left(\overline{\varphi_{i}} f\right)\right\|_{\mathbb{A}_{\alpha}^{2}} \leq\left\|\varphi_{i} f\right\|_{\mathbb{A}_{\alpha}^{2}}$ and then

$$
\sum_{i=1}^{l}\left\|B_{\alpha}\left(\overline{\varphi_{i}} f\right)\right\|_{\mathbb{A}_{\alpha}^{2}} \leq \sup _{\mathbb{B}}|\Phi(w)|\|f\|_{\mathbb{A}_{\alpha}^{2}} .
$$

Let $f_{w}(z)=\frac{B_{\alpha}(z, w)}{\sqrt{B_{\alpha}(w, w)}}$, with $w$ in $\mathbb{B}$. Then $H f_{w}(z)=\sum_{i=1}^{l} \varphi_{i}(z) \bar{\varphi}_{i}(w) f_{w}(z)$ and $\left\langle H f_{w}, f_{w}\right\rangle_{\alpha}=|\Phi(w)|^{2}$, where $\langle\cdot, \cdot\rangle_{\alpha}$ is the inner product of $\mathbb{A}_{\alpha}^{2}$. The relation $\left\langle H f_{w}, f_{w}\right\rangle_{\alpha} \leq\|H\|\|f\|_{\mathbb{A}_{\alpha}^{2}}^{2}$ and the fact that $\left\|f_{w}\right\|_{\mathbb{A}_{\alpha}^{2}}=1$ finish the proof. 
Proof of Theorem 1.1. Assume that $\sup _{\mathbb{B}}|\Phi(w)|^{2} \leq 1$. Then $H$ is a contraction and the operator $(I-H)^{1 / 2}$ is a well defined positive operator. It is well known that $(I-H)^{1 / 2}\left(\mathbb{A}_{\alpha}^{2}\right)$ is a sub-Hilbert space with reproducing kernel given by $(I-H) B_{\alpha}(z, w)=K_{\alpha}(z, w)$. See [8] and [10] for details.

Assume now that $K_{\alpha}(z, w)$ is a reproducing kernel; then for any finite sequence $\left(w_{j}\right)_{j=1 . . m}$ of points of the ball, the matrix $\left(K_{\alpha}\left(w_{j}, w_{k}\right)\right)_{j, k=1 . . m}$ is positive definite [1]. If we consider a point $w$ in the ball, the positivity gives $K_{\alpha}(w, w)=$ $\left(1-|\Phi(w)|^{2}\right) B_{\alpha}(w, w) \geq 0$, and then $|\Phi(w)|^{2} \leq 1$.

Proof of Theorem 1.2. The proof involves the Douglas criterion [4]; we give here the statement.

Douglas criterion: Let $\mathcal{H}_{1}, \mathcal{H}_{2}$ and $\mathcal{H}$ be Hilbert spaces. Let $A$ from $\mathcal{H}_{1}$ into $\mathcal{H}$ and $B$ from $\mathcal{H}_{2}$ into $\mathcal{H}$ be bounded operators.

There exists $\lambda>0$ such that $A A^{*} \ll \lambda B B^{*}$ if and only if $\operatorname{Im}(A) \subset \operatorname{Im}(B)$.

In the definition, the relation $A A^{*} \ll \lambda B B^{*}$ means that $\lambda B B^{*}-A A^{*}$ is a positive operator.

We consider the identity map $\Phi_{0}(z)=z$ and we denote by $H_{0}$ its associated operator given by Definition 2.1. The kernel of the Hilbert space $\left(I-H_{0}\right)^{1 / 2}\left(\mathbb{A}_{\alpha}^{2}\right)$ is $(1-z \bar{w}) B_{\alpha}(z, w)=B_{\alpha-1}(z, w)$. Then $\left(I-H_{0}\right)^{1 / 2}\left(\mathbb{A}_{\alpha}^{2}\right)=\mathbb{A}_{\alpha-1}^{2}$ by the uniqueness of the reproducing kernel.

If we suppose that $K_{\alpha}(z, w)$ is a reproducing kernel of $\mathbb{A}_{\alpha-1}^{2}$, then by the Douglas criterion there exists $\lambda>0$ such that $I-H \ll \lambda\left(I-H_{0}\right)$. Let $f$ be in $\mathbb{A}_{\alpha}^{2}$. Then $\langle(I-H) f, f\rangle_{\alpha} \leq \lambda\left\langle\left(I-H_{0}\right) f, f\right\rangle_{\alpha}$. Take $w$ in $\mathbb{B}$. The function $f_{w}(z)=B_{\alpha}(z, w)$ gives $1-|\Phi(w)|^{2} \leq \lambda^{\prime}(-r(w))$.

In the case where $\alpha \geq 1$, the sufficient condition follows from the next result:

Proposition 2.3. Let $\Phi(z)=\left(\varphi_{1}(z), \cdots, \varphi_{l}(z)\right)$ be holomorphic. If $1-|\Phi(z)|^{2} \leq$ $C(-r(z))$, there exists $\lambda>0$ such that

$$
\frac{1}{\lambda}\left(I-H_{0}\right) \ll I-H \ll \lambda\left(I-H_{0}\right) .
$$

Proof of Proposition 2.3. Let $\alpha \geq 1$. The right "inequality" follows from two technical lemmas:

Lemma 2.4. There exists $C>0$ such that for $f$ in $\mathbb{A}_{\alpha}^{2}$,

$$
\langle(I-H) f, f\rangle_{\alpha} \leq C\|f\|_{\mathbb{A}_{\alpha+1}^{2}}^{2} .
$$

Proof. Take $f$ in $\mathbb{A}_{\alpha}^{2}$. Then

$$
\langle(I-H) f, f\rangle_{\alpha}=\int_{\mathbb{B}} \int_{\mathbb{B}} K_{\alpha}(z, w) f(w) \overline{f(z)}(-r(w))^{\alpha}(-r(z))^{\alpha} d V(w) d V(z) .
$$

Let $K(z, w)=K_{\alpha}(z, w)(-r(w))^{-1 / 2+\alpha / 2}(-r(z))^{-1 / 2+\alpha / 2}$ and denote by $K$ the integral operator given by

$$
K g(z)=\int_{\mathbb{B}} K(z, w) g(w) d V(w), g \in L^{2}(\mathbb{B}) .
$$


Assume for the moment that $K$ is bounded in $L^{2}(\mathbb{B})$. We consider the function $g(z)=f(z)(-r(z))^{1 / 2+\alpha / 2}$; then

$$
\begin{aligned}
\langle(I-H) f, f\rangle_{\alpha} & =\int_{\mathbb{B}} K g(z) \overline{g(z)} d V(z) \\
& \leq\|K(g)\|_{L^{2}(\mathbb{B})}\|f\|_{\mathbb{A}_{\alpha+1}^{2}} \leq C\|f\|_{\mathbb{A}_{\alpha+1}^{2}}^{2} .
\end{aligned}
$$

It remains to study the boundedness of $K$. This follows from the Schur criterion [12]. We only have to prove that there exists $C>0$ such that $|K(z, w)| \leq \frac{C}{|1-z \bar{w}|^{n+1}}$. Recall that (see [6, Prop. 8.1.4])

$$
\frac{|1-\Phi(z) \overline{\Phi(w)}|^{2}}{\left(1-|\Phi(z)|^{2}\right)\left(1-|\Phi(w)|^{2}\right)} \leq \frac{|1-z \bar{w}|^{2}}{(-r(z))(-r(w))} .
$$

Therefore there exists $C>0$ such that $|1-\Phi(z) \overline{\Phi(w)}| \leq C|1-z \bar{w}|$, and then $|K(z, w)|$ has the desired pointwise estimate.

We finish the proof of the proposition with the second technical lemma:

Lemma 2.5. Let $\alpha>-1$. There exists $C>0$ such that

$$
\frac{1}{C}\|f\|_{\mathbb{A}_{\alpha+1}^{2}}^{2} \leq\left\langle\left(I-H_{0}\right) f, f\right\rangle_{\alpha} \leq C\|f\|_{\mathbb{A}_{\alpha+1}^{2}}^{2}, f \in \mathbb{A}_{\alpha}^{2} .
$$

Proof. The proof is direct. Let $f(z)=\sum_{m} a_{m} z^{m}$. Recall that

$$
\left\|z^{m}\right\|_{\mathbb{A}_{\alpha}^{2}}^{2}=c_{\alpha} \frac{\Gamma\left(m_{1}+1\right) \cdots \Gamma\left(m_{n}+1\right)}{\Gamma(|m|+n+1+\alpha)},
$$

where $|m|=m_{1}+\cdots+m_{n}$ [6]. Let us remark that $(1-z \bar{w}) B_{\alpha}(z, w)=c B_{\alpha-1}(z, w)$. Then

$$
\left(I-H_{0}\right) f(z)=c \int_{\mathbb{B}} B_{\alpha-1}(z, w) f(w)(-r(w))^{\alpha} d V(w)=c \sum_{m} \frac{a_{m} z^{m}}{|m|+n+\alpha}
$$

and

$$
\left\langle\left(I-H_{0}\right) f, f\right\rangle_{\alpha}=c \sum_{m}\left|a_{m}\right|^{2} \frac{\Gamma\left(m_{1}+1\right) \cdots \Gamma\left(m_{n}+1\right)}{(|m|+n+\alpha) \Gamma(|m|+n+1+\alpha)} .
$$

Notice that

$$
\|f\|_{\mathbb{A}_{\alpha+1}^{2}}^{2}=c \sum_{m}\left|a_{m}\right|^{2}\left\|z^{m}\right\|_{\mathbb{A}_{\alpha+1}^{2}}^{2}=c \sum_{m}\left|a_{m}\right|^{2} \frac{\Gamma\left(m_{1}+1\right) \cdots \Gamma\left(m_{n}+1\right)}{\Gamma(|m|+n+2+\alpha)} .
$$

The equivalence follows from the relation $\Gamma(z+1)=z \Gamma(z)$.

For the left "inequality" of Proposition [2.3, we consider the auxiliary operator $\widetilde{H}$ given by $\widetilde{H} f=T_{|\Phi|^{2}}(f)=B_{\alpha}\left(|\Phi|^{2} f\right), \alpha>-1$. Let us remark that $\|\widetilde{H}\| \leq \sup _{\mathbb{B}}|\Phi(w)|^{2}$ and $\langle H f, f\rangle_{\alpha}=\sum_{i=1}^{l}\left\langle\varphi_{i} f, B_{\alpha}\left(\varphi_{i} f\right)\right\rangle_{\alpha} \leq \sum_{i=1}^{l}\left\langle\varphi_{i} f, \varphi_{i} f\right\rangle_{\alpha}=$ $\langle\widetilde{H} f, f\rangle_{\alpha}$; then

$$
I-\widetilde{H} \ll I-H
$$

We have the following result:

Lemma 2.6. Let $\widetilde{H}$ be as above. There exists $\lambda>0$ such that $I-H_{0} \ll \lambda(I-\widetilde{H})$. 
Proof. Let us remark that $1-|\Phi(0)|^{2} \leq 2|1-\Phi(z) \overline{\Phi(0)}|$. It follows from the relation (2.3) that there exists $C>0$ such that $-r(z) \leq C\left(1-|\Phi(z)|^{2}\right)$ and $\|f\|_{\mathbb{A}_{\alpha+1}^{2}}^{2} \leq$ $C\langle(I-\widetilde{H}) f, f\rangle_{\alpha}$. It remains to apply Lemma 2.5 to achieve the proof.

The proof is similar for $-1<\alpha \leq 1$, but a minor modification is required to use the Schur criterion. We recall that for holomorphic functions $u$ and $v[6$,

$$
\begin{aligned}
\int_{\mathbb{B}} u(w) \overline{v(w)}(-r & (w))^{\alpha} d V(w) \\
& =\int_{\mathbb{B}}(R+(n+1+\alpha) I) u(w) \overline{v(w)}(-r(w))^{\alpha+1} d V(w) \\
& =\int_{\mathbb{B}} u(w)(\bar{R}+(n+1+\alpha) I) \overline{v(w)}(-r(w))^{\alpha+1} d V(w) .
\end{aligned}
$$

The kernel of $\left(I-H_{0}\right)^{1 / 2}\left(\mathbb{A}_{\alpha}^{2}\right)$ is given by $(1-z \bar{w}) B_{\alpha}(z, w)=c B_{\alpha-1}(z, w)$; thus $\left(I-H_{0}\right)^{1 / 2}\left(\mathbb{A}_{\alpha}^{2}\right)$ is the weighted Bergman space $\mathbb{A}_{\alpha-1}^{2}$ when $0<\alpha<1$, the Hardy space $\mathbb{H}^{2}$ for $\alpha=0$ and the weighted Besov space $\mathcal{B}_{\alpha-1}$ for $-1<\alpha<0$.

For $f$ in $\mathbb{A}_{\alpha}^{2}$,

$$
(I-H) f(z)=\int_{\mathbb{B}}(\bar{R}+(n+1+\alpha) I) K_{\alpha}(z, w) f(w)(-r(w))^{\alpha+1} d V(w)
$$

and the relation (2.1) becomes

$$
\langle(I-H) f, f\rangle_{\alpha}=\int_{\mathbb{B}}(R+(n+1+\alpha) I)(I-H) f(z) \overline{f(z)}(-r(z))^{\alpha+1} d V(z) .
$$

Let

$$
\begin{aligned}
K(z, w)=(- & r(z))^{1 / 2+\alpha / 2}(-r(w))^{1 / 2+\alpha / 2} \\
& \times(R+(n+1+\alpha) I)(\bar{R}+(n+1+\alpha) I) K_{\alpha}(z, w)
\end{aligned}
$$

and let $K$ be the integral operator given by (2.2). Then

$$
\langle(I-H) f, f\rangle_{\alpha}=\int_{\mathbb{B}} K g(z) \overline{g(z)} d V(z)
$$

with $g(z)=f(z)(-r(z))^{1 / 2+\alpha / 2}$. Let us remark that

$$
\begin{aligned}
K(z, w)=\sum_{s, t=0}^{1}(I+ & (n+1) R)^{s}(I+(n+1) \bar{R})^{t}(1-\Phi(z) \overline{\Phi(w)}) \\
& \times(I+(n+1) R)^{1-s}(I+(n+1) \bar{R})^{1-t} B_{\alpha}(z, w) .
\end{aligned}
$$

Then there exists $C>0$ such that

$$
\begin{aligned}
&|K(z, w)| \leq C(-r(z))^{1 / 2+\alpha / 2}(-r(w))^{1 / 2+\alpha / 2}\left(\frac{|1-\Phi(z) \overline{\Phi(w)}|}{|1-z \bar{w}|^{n+1+\alpha+2}}\right. \\
&\left.+\frac{\sum_{i}\left|\frac{\partial \varphi_{i}(w)}{\partial w_{j}}\right|+\sum_{i}\left|\frac{\partial \varphi_{i}(z)}{\partial z_{j}}\right|}{|1-z \bar{w}|^{n+1+\alpha+1}}+\frac{\sum_{i}\left|\frac{\partial \varphi_{i}(w)}{\partial w_{j}}\right|\left|\frac{\partial \varphi_{i}(z)}{\partial z_{j}}\right|}{|1-z \bar{w}|^{n+1+\alpha}}\right) .
\end{aligned}
$$


Recall that $|1-\Phi(z) \overline{\Phi(w)}| \leq C|1-z \bar{w}|$ and let us remark that the condition on $\Phi$ gives $\sup _{\mathbb{B}}\left|\frac{\partial \varphi_{i}(w)}{\partial w_{j}}\right| \leq C$; then

$$
|K(z, w)| \leq \frac{C(-r(z))^{\alpha / 2+1 / 2}(-r(w))^{\alpha / 2+1 / 2}}{|1-z \bar{w}|^{n+\alpha+2}} .
$$

Let us remark that $(-r(z))$ and $(-r(w))$ are bounded by a constant times $|1-z \bar{w}|$. Then $|K(z, w)| \leq \frac{C}{|1-z \bar{w}|^{n+1}}$. The operator $K$ is bounded in $L^{2}(\mathbb{B})$ and the relation (2.1) gives $\langle(I-H) f, f\rangle_{\alpha} \leq C\|f\|_{\mathbb{A}_{\alpha+1}^{2}}^{2}$. Lemma 2.5 gives $I-H \leq \lambda(I-$ $\left.H_{0}\right)$.

Theorem 1.2 characterizes the sub-Hilbert space $(I-H)^{1 / 2}\left(\mathbb{A}_{\alpha}\right)$ : this space is the analogue of the unit ball of $\mathbb{C}^{n}$ for the complementary space [3]. We consider the space $(I-\widetilde{H})^{1 / 2}\left(\mathbb{A}_{\alpha}\right)$; it is a consequence of the relation (2.4) and Lemma 2.6 that there exists $C>0$ such that

$$
\frac{1}{C}\left(I-H_{0}\right) \ll I-\widetilde{H} \ll I-H .
$$

Under the condition on $\Phi$ given in Theorem 1.2, we have

$$
(I-\widetilde{H})^{1 / 2}\left(\mathbb{A}_{\alpha}\right)=(I-H)^{1 / 2}\left(\mathbb{A}_{\alpha}\right) .
$$

\section{REFERENCES}

1. N. Aronszajn, Theory of reproducing kernels, Trans. Amer. Math. Soc. 68 (1950), 337-404. MR0051437 (14:479c)

2. F. Beatrous and J. Burbea, Positive-definiteness and its applications to interpolation problems for holomorphic functions, Trans. Amer. Math. Soc. 284 (1984), 247-270. MR742424 (85e:32020)

3. L. de Branges and J. Rovnyak, Square summable power series, Holt, Rinehart and Winston, New York, 1966. MR0215065 (35:5909)

4. R. Douglas, On majorization, factorization, and range inclusion of operators on Hilbert space, Proc. Amer. Math. Soc. 1 (1966), 413-415. MR0203464 (34:3315)

5. S. Krantz, Function theory of several complex variables, John Wiley and Sons, New York, 1982. MR635928 (84c:32001)

6. W. Rudin, Function theory in the unit ball of $\mathbb{C}^{n}$, Classics in Mathematics 241, SpringerVerlag, New York, 1980. MR601594 (82i:32002)

7. S. Saitoh, Theory of reproducing kernels and its applications, Pitman Research Notes in Mathematics 189, Wiley, New York, 1988. MR983117 (90f:46045)

8. D. Sarason, Sub-Hardy Hilbert spaces in the unit disk, John Wiley \& Sons, Inc., New York, 1994. MR 1289670 (96k:46039)

9. S. Sultanic, Sub-Bergman Hilbert spaces, J. of Math. Anal. Appl. 234 (2005), 639-649. MR:2262497 (2007h:46042)

10. K. Zhu, Sub-Bergman Hilbert spaces on the unit disk, Indiana Univ. Math. J. 45 (1996), 165-176. MR.1406688 (97f:46043)

11. K. Zhu, Sub-Bergman Hilbert spaces on the unit disk II, Journal of Funct. Anal. 202 (2003), 327-341. MR.1990528 (2004g:46040) 
12. K. Zhu, Operator theory in function spaces, Marcel Dekker, New York, 1990. MR 1074007 (92c:47031)

13. K. Zhu, Spaces of holomorphic functions in the unit ball, Springer-Verlag, New York, 2004. MR 2115155 (2006d:46035)

Laboratoire de Mathématiques et Application, Université de Poitiers, UMR 6086 CNRS, Téléport 2, Boulevard Pierre et Marie Curie, BP30179, 86962 Futuroscope, FRANCE

E-mail address: frederic.symesak@univ-poitiers.fr 